\title{
Versorgung von mechanisch und hämodynamisch instabilen Beckenverletzungen
}

\author{
Pol Maria Rommens, Jens Heinermann, Martin Henri Hessmann
}

\section{Zusammenfassung}

Retroperitoneale Blutungen als Folge einer Ruptur des hinteren Beckenringes können schnell zum Verblutungstod führen, wenn sie nicht erkannt und zeitgerecht behandelt werden. Die klinischen Untersuchung der Stabilität und des umgebenden Weichteilmantels sowie Röntgendiagnostik - mit Beckenübersichtsaufnahme und Computertomographie als absolutes Minimum - geben Hinweise über Art und Ausdehnung der Instabilität und der Weichteilschäden. Die ersten Maßnahmen entsprechen dem ABC des ATLS. Weiterhin kommen äußere Kompression, offene Tamponade, Arteriographie und Embolisation und chirurgische Hemipelvekto- mie in Frage. Die äußere Kompression ist die zuerst empfohlene Behandlung. Bei lebensbedrohlicher Blutung wird die Beckenzwinge eingesetzt, bei hämodynamischer Instabilität der Fixateur externe. Beide Systeme müssen als temporäre Lösungen angesehen werden und haben ihre spezifische Gefahren und Beschränkungen. Die Wahl der definitiven Behandlung der Beckenringinstabilität richtet sich nach verschiedenen Kriterien. Die Beckenringverletzung vom Typ $\mathrm{C}$ ist gefährlicher als die vom Typ B. Dies wird durch eine höhere Mortalität sowie durch eine höhere Zahl an begleitenden Weichteilverletzungen, die zu schlechteren funktionellen Endergebnissen führen, deutlich.

Wenige akut lebensbedrohliche Situationen nach Unfall sind direkt auf Verletzungen des muskuloskelettalen Systems zurückzuführen. Schwerste Schädelhirntraumen, Lungenkontusionen und intraperitoneale Blutungen oder die Ruptur von großen Gefäßen sind eher für das frühe Sterben nach Trauma verantwortlich als Wirbelfrakturen am thorakolumbalen Übergang, Oberschenkelfrakturen oder drittgradig offene Unterschenkelfrakturen. Die komplexe Beckenringfraktur stellt neben den Mittelgesichtsfrakturen mit Verlegung der Atemwege eine Ausnahme dar. Retroperitoneale Blutungen als Folge einer Ruptur des hinteren Beckenringes können schnell zum Verblutungstod führen, wenn sie nicht erkannt und zielgerecht behandelt werden.

OP-JOURNAL 2003; 19: 110-116

(c) Georg Thieme Verlag Stuttgart · New York
Bltungen aus dem hinteren Beckenring können zum Verblutungstod führen, wenn sie nicht zeitlich erkannt und sachgerecht behandelt werden.

In diesem Manuskript werden die Prioritäten sowie die Möglichkeiten und Grenzen verschiedener stabilisierender Maßnahmen für das instabile Becken vorgestellt.

\section{Primärdiagnostik}

Viele schwerverletzte Patienten weisen eine Beckenringverletzung auf, viele Patienten mit einer Beckenringverletzung sind schwerverletzt. Es gehört deshalb zu den ersten Aufgaben des aufnehmenden Arztes, bereits im Schockraum eine Beckenringverletzung als Blutungsquelle auszuschließen. Zur Primärdiagnostik gehört eine Prüfung der Beckenstabilität sowie eine klinische Prüfung der Weichteile um den Beckengürtel herum und des Perineums. Wenn beide Beckenschaufeln zueinander bewegt werden können, besteht eine rotatorische Instabilität. Wenn eine Beckenhälfte durch Längszug nach distal bewegt werden kann, besteht eine vertikale Instabilität. Offene Verletzungen des Beckenringes oder Pfählungsverletzungen mit Perforation der inneren Organe haben einen sehr negativen Einfluss auf die Gesamtprognose. Die Beckenübersichtsaufnahme gehört zusammen mit der Thoraxübersichtsaufnahme und die laterale Aufnahme der zervikalen Wirbelsäule zu den konventionellen Röntgenaufnahmen, die bei allen polytraumatisierten Patienten sofort nach Klinikaufnahme ausnahmslos angefertigt werden. Damit eine Röntgenaufnahme von guter Qualität erreicht wird, muss der Patient entkleidet werden und auf dem Rücken liegen. Der Röntgenstrahl soll senkrecht auf die Symphysis pubica gerichtet sein. Wenn anhand dieser Röntgenaufnahme eine Beckenringverletzung ausgeschlossen wurde, darf auf weitere Diagnostik des Beckenringes verzichtet werden. Bei Verdacht auf eine Verletzung oder falls eine Beckenringverletzung auf der Beckenübersichtsaufnahme bestätigt wird, sollen weitere Informationen zu der Identität dieser Verletzung gewonnen werden. Wenn die hämodynamische Situation des Patienten dies zulässt, werden zwei weitere Röntgenbilder genommen. Dies sind die Beckeneingangsund Beckenausgangsaufnahme. Sie erlauben eine bessere Einschätzung der Art der Instabilität sowie die Identifikation des Ausmaßes und der Richtung der Dislokation der Beckenringfragmente. Wenn der Allgemeinzustand des Patienten einen Transport in die Röntgenabteilung ermöglicht, wird auch eine CT-Untersuchung des Beckens durchgeführt. Sie ist für die Feindiagnostik und das Festlegen des Behandlungsmodus unerlässlich. Zunehmend wird mit Spiral-CT-Geräten in der Frühphase nach Klinikaufnahme ein Ganzkörper CT angefertigt. Diese CT-Geräte der jüngsten Generation erlauben es vier, manchmal sogar 16 Schichten parallel aufzunehmen. 


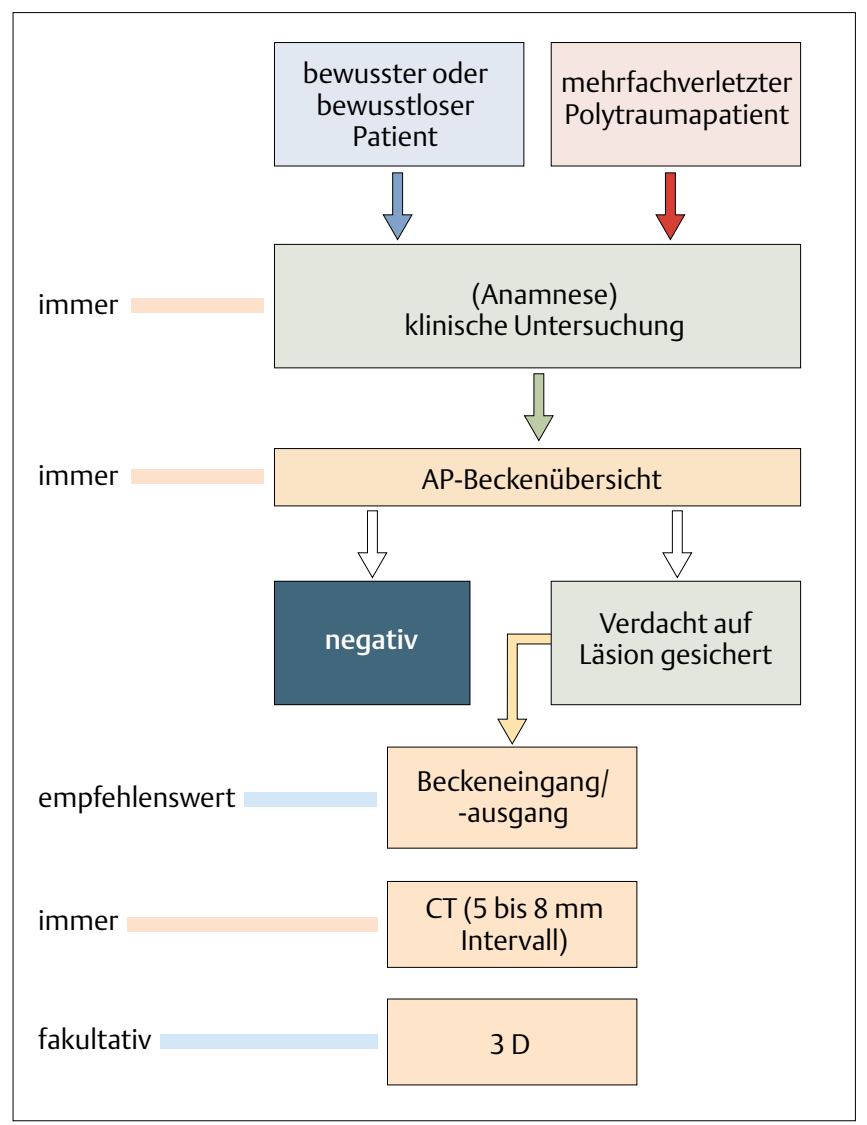

Abb. 1 Entscheidungsdiagramm zur radiologischen Diagnostik einer Beckenringverletzung.
Die Beckenübersichtsaufnahme ist als orientierende Untersuchung bei jedem schwerverletzten Patienten unverzichtbar. Sie wird durch eine computertomographische Untersuchung zur genauen Beurteilung des hinteren Beckenringes ergänzt.

Das so gewonnene Datenmaterial dient als umfassende und orientierende Untersuchung während der Erstversorgung von Schwerverletzten. Während Art und Lokalisation einer Verletzung sofort erkannt werden, erfordert die detaillierte Auswertung der Daten mehr Zeit. Zweiund dreidimensionale Rekonstruktionen der CT-Bilder stellen ebenfalls keine Priorität dar. Für die Planung der definitiven Chirurgie und das Festlegen des Osteosyntheseverfahrens des vorderen und hinteren Beckenringes sind sie jedoch sehr hilfreich (Abb. 1) [4].

\section{Hämodynamische Instabilität}

Die primäre Behandlung von Patienten mit einer Beckenringverletzung wird sich - wie bei schwerverletzten Patienten ohne Beckenringverletzung - nach der hämodynamischen Situation und nicht nach der Art der mechanischen Instabilität richten. Der kritische hämodynami- sche Zustand des Patienten wird durch Blutverlust erklärt. Dieser Blutverlust ist vor allem venöser Art und stammt aus den Frakturfragmenten des hinteren Beckenringes. Weiterhin sind viele kleine Gefäße aus dem Plexus präsakralis und aus dem Beckenboden rupturiert. Viel weniger häufig - geschätzt wird eine Frequenz von unter 10 von 100 - sind Blutungen aus Arteriolen oder Arterien wie die A. obturatoria oder A. glutea superior für einen kontinuierlichen Blutverlust verantwortlich. Der Blutverlust stammt deshalb in der Regel von dem Niedrigdrucksystem und könnte darum theoretisch leicht durch direkte Kompression zum Stillstand gebracht werden.

Das ABC des ATLS gilt als Leitlinie der Wiederbelebung eines hämodynamisch instabilen Patienten mit Beckenringverletzung

Die ersten Maßnahmen zur Wiederbelebung eines Schwerverletzten mit Beckenringtrauma entsprechen dem $A B C$ des ATLS. Die Atemwege des Patienten werden zuerst sichergestellt. In der Regel ist eine Intubation indiziert. Weiterhin wird auf eine ausreichende periphere Gewebeoxygenierung geachtet. Raumluft wird mit Sauerstoff angereichert. Nach Intubation erfolgt eine mechanische Ventilation. Es folgt eine aggressive Volumensubstitution mit kolloiden und kristalloiden Lösungen. Schnellstmöglich werden Plasma sowie kompatible Erythrozytenkonzentrate verabreicht.

Als lebensrettende Maßnahme wird das instabile Becken von außen komprimiert. Dieser Aspekt wird in einem separaten Teil dieses Manuskriptes näher erläutert.

Weitere Möglichkeiten sind die selektive Arteriographie und Embolisation, die offene Tamponade und die chirurgische $\mathrm{He}$ mipelvektomie. Die selektive Arteriographie und Embolisation haben ein nur beschränktes Spektrum von Indikationen, weil die arterielle Blutung bei nur wenigen Patienten für das hämodynamische Bild des Patienten verantwortlich ist. Wir sehen eine Indikation nur gegeben, wenn nach aggressiver Volumensubstitution und nach äußerer Beckenkompression keine hämodynamische Stabilität erreicht werden konnte und eine andere Blutungsquelle (thorakal, abdominal) ausgeschlossen wurde. Die Angiographie ist stets mit einem hohen technischen Aufwand und mit einer entsprechend langen Vorbereitungszeit verbunden. Es ist gefährlich, einen hämodynamisch instabilen Patienten ohne jegliche Form von Beckenringstabilisierung in den Angiographieraum zu transportieren. Dort fehlt die Logistik des Schockraumes, wichtige Zeit und Möglichkeiten zur Behandlung von nicht-arteriellen Blutungen könnten verloren gehen [1].

Selektive Arteriographie und Embolisation ist nur sinnvoll, wenn die Blutung aus dem instabilen Beckenring von arteriellem Ursprung ist.

Die bessere Alternative zur Arteriographie ist die offene Tamponade durch Packing. Weil sich die Blutungsquelle im kleinen Becken befindet, das mit dem Retroperitoneal- und nicht mit dem Abdominalraum verbunden ist, erübrigt sich das Eröffnen des Abdomens. Selbstverständlich sollen nachgewiesene intraabdominelle Blutungen durch Laparotomie angegangen werden. Der optimale Zugang zum kleinen Becken ist der mediane infraumbilikale Längsschnitt. Bei Bedarf kann er nach proximal erweitert werden. Als Alternative gilt der Pfannenstielschnitt. Er wird nur benutzt, wenn auf eine Laparotomie verzichtet werden kann. Der Raum hinter der Symphysis pubica - das Spatium Retzii - wird 


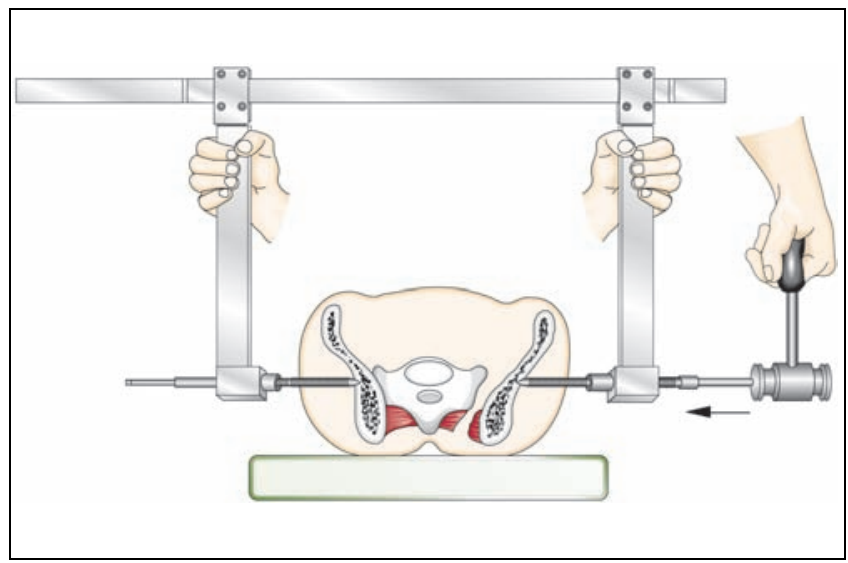

Abb.2a Beckenzwinge bei Platzierung der beiden Schanz'schen Schrauben auf der äußeren Kortikalis des dorsalen Iliums.

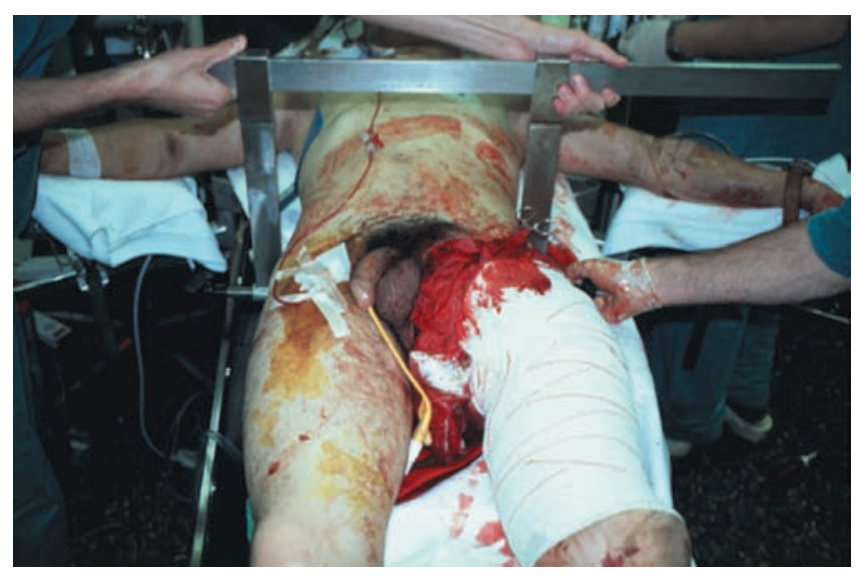

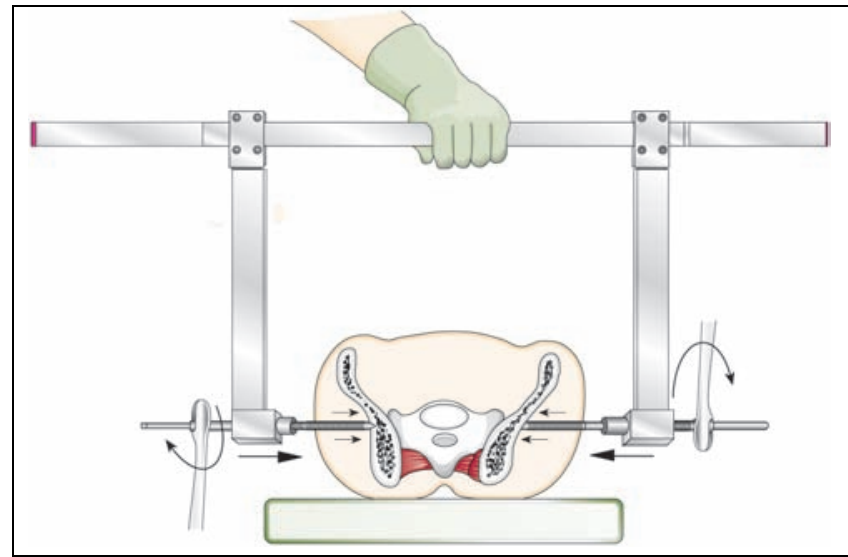

Abb.2b Verklemmung der beiden Beckenhälften durch äußere Kompression.

Abb. 2c Klinisches Beispiel einer Beckenzwinge, die am hämodynamisch instabilen Patienten nach Überrolltrauma angelegt wurde. aufgesucht und geöffnet. In der Regel findet sich hier ein großes, wachsendes Hämatom, das vorsichtig entfernt wird. Nun wird der freigewordene Raum mit sterilen Tüchern oder Kompressen gefüllt. Die Schnittführung wird temporär verschlossen. Wenn vorher eine Beckenzwinge oder ein Fixateur externe angelegt wurde, entsteht im kleinen Becken eine wirksame Tamponade durch Dichtdrücken der kleineren Gefäße. Wenn der Beckenring nicht stabilisiert wurde, werden die instabilen Beckenhälften durch das Packing weiter auseinander getrieben.

Die chirurgische Dekompression des Hämatoms mit Tamponade des kleinen Beckens ist zwar eine invasive, aber sehr effiziente Maßnahme zur Stillung einer aktiven Blutung aus dem instabilem Beckenring. Sie setzt eine äußere Kompression des Beckenringes voraus.

Die hämodynamische Effektivität des Packings muss durch Monitoring schnell erkennbar werden. Wenn dies der Fall ist, kann der Patient für 48 Stunden auf der
Intensivstation aufgenommen werden. Während dieser Zeit werden seine Vitalparameter - systolischer Blutdruck, Gewebeoxygenierung, Hämoglobin und Hämatokritwert, Urinausscheidung sowie Blutgerinnung - optimiert. Danach kehrt er in den Operationssaal zurück. Während der Second-look-Chirurgie werden die Tücher oder Kompressen vorsichtig entfernt und der freigewordene Raum ausgiebig gespült und drainiert. Noch vorhandene kleinere Blutungen werden individuell durch Übernähen gestillt $[2,5]$.

Die chirurgische Hemipelvektomie ist die allerletzte Möglichkeit, das Leben des schwerverletzten Patienten zu retten. Sie wird nur bei einer bereits traumatisch stattgehabten Hemipelvektomie angewandt. Diese kann geschlossen oder offen, komplett oder inkomplett sein. Eine traumatische Hemipelvektomie ist durch eine unilaterale oder bilaterale komplette mechanische Instabilität des vorderen und hinteren Beckenringes gekennzeichnet. Darüber hinaus besteht eine Ruptur oder ein Verschluss der wichtigsten pelvinen Gefäße, und die Nervenbahnen sind ausgerissen oder überdehnt. Die Muskeln sind vom Beckenring teilweise oder komplett getrennt und die Haut sowie das subkutane Fettgewebe sind von den unterliegenden Strukturen losgelöst und/oder rupturiert. Als erste Maßnahme wird die stets vorhandene Blutung durch manuelle Kompression gestillt. Es folgt ein schnellstmöglicher Transport ins Krankenhaus, wo die Hemipelvektomie nur komplettiert werden kann. Aus der Literatur ist bekannt, dass Erhaltungsversuche das Überleben des Patienten gefährden. Die Wunde wird ausgiebig gespült, gereinigt und alle Blutungen durch Übernähen gestoppt. Begleitende Weichteilverletzungen wie Blasenruptur, Rektum oder Vaginaeinriss oder Urethraruptur werden einzeln versorgt. Die Wunde wird offen gelassen und in weiteren Eingriffen gereinigt und progressiv verkleinert [6].

\section{Mechanische Instabilität}

Neben den oben genannten Maßnahmen kann der Chirurg die vorhandene Instabilität durch direkte Kompression auf den Beckenring verringern. Das Ziel dieser äußeren Beckenkompression ist nicht die Wiederherstellung der Anatomie des vorderen oder hinteren Beckenringes, sondern die Wiederherstellung der Stabilität im kleinen Becken, damit eine (Selbst) Tamponade durch Koagulation ermöglicht wird. Die Mittel, die dafür zur Verfügung stehen, sind die Beckenzwinge und der Fixateur externe. Viel weniger effektiv ist das Komprimieren mit einem Bettlaken, das um das instabile Becken gewickelt wird. Im Notartwagen wird zur Zeit ein Beckengürtel aus Textil erprobt. Er besteht aus verschiedenen aufblasbaren Kammern, die einen hohen lokalen Druck entwickeln. Die Beckenzwinge ist ein In- 


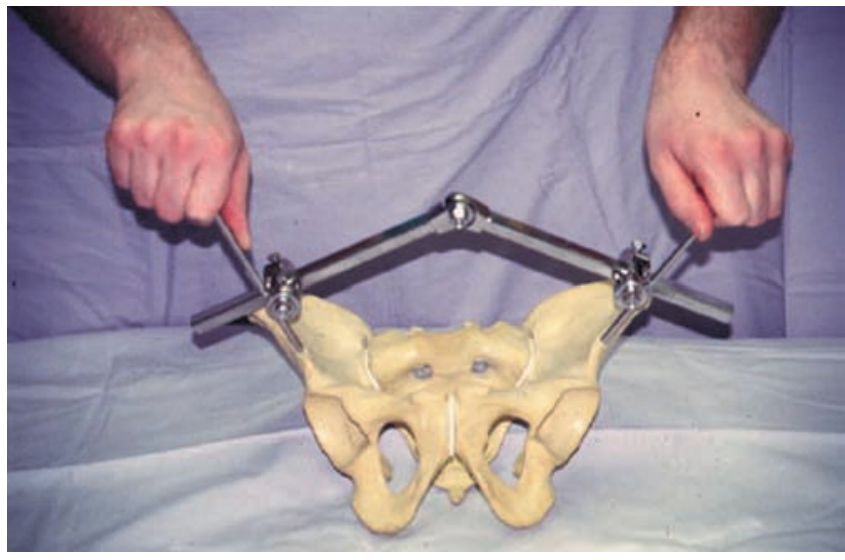

Abb. 3a Brückenfixateur: Eine Schanz'sche Schraube supraacetabulär auf beiden Seiten wird mit zwei Fixateurstangen in Form einer Brücke verbunden.

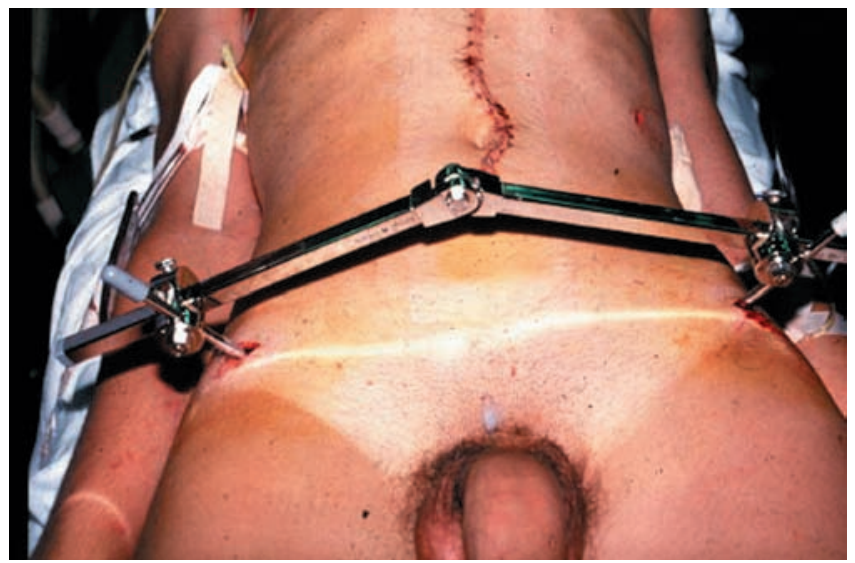

Abb.3b Klinisches Beispiel.
Abb.3d CT-Schnitt supraacetabulär bei $\mathrm{Pa}$ tienten mit liegendem Brückenfixateur.

strument, das bei Patienten mit einer lebensbedrohlichen Blutung aus dem instabilen Becken eingesetzt wird. Zwei Schanz'sche Schrauben, fest verbunden mit einer C-förmigen Klemme drücken die gebrochenen Beckenhälften aufeinander. Die Schanz'schen Schrauben werden durch Stichinzisionen und durch die Glutealmuskulatur auf die äußere
Kortikalis des dorsalen Iliums in Höhe des Sakroiliakalgelenkes gedrückt. Auf Grund der Dringlichkeit wird die Beckenzwinge bereits im Schockraum unter semi-sterilen Notfallbedingungen am Becken angebracht. Hierzu ist die Mitarbeit von zwei Kollegen, die je an einer Seite des verletzten Beckens aufgestellt sind, erforderlich (Abb.2a-c). Ganz et al. konnten deutlich zeigen, dass die Kompression des hinteren Beckenringes zu einer sofortigen Abnahme des Blutverlustes und somit zu einer schnell messbaren Zunahme des systolischen Blutdruckes der betroffenen Patienten führte [3].

Die Beckenzwinge drückt das auseinandergebrochene Becken am hinteren Beckenring wieder zusammen. Durch die gewonnene Stabilität wird eine Selbsttamponade möglich.

Die Platzierung der Schanz'schen Schrauben in einer Notfallsituation bringt erhebliche Gefahren mit sich. Bei zu hoher Kompression können die Schrauben die Kortikalis perforieren und mit der Spitze im Sakrumkörper zu liegen kommen. Bei zu hoher oder zu niedriger Anlage der Schrauben kommen die Spitzen in der Rückenmuskulatur oder durch die Incisura ischiadica major im Beckeninneren zu liegen. Wenn die Instabilität durch eine Iliumfraktur und nicht durch eine Sakrumfraktur oder sakroiliakale Luxation erklärt wird, dann wird die Beckenzwinge keine Stabilität herbeiführen können. Auch wird beanstandet, dass eine zu hohe Kompression auf die Beckenhälften bei einer transforaminalen Sakrumfraktur zu Kompressionsschädigung der sakralen Wurzeln führen könnte. Es ist deshalb wichtig, die Beckenzwinge vor ihrem Einsatz einmal detailliert anzusehen und ihre Wirkung an einem Beckenmodell auszuprobieren.

Das Anbringen der Beckenzwinge ist eine Notfallmaßnahme. Die Beckenzwinge wird nicht als eine definitive Behandlung der Beckeninstabilität betrachtet. Bei Überleben des Patienten und Wiederherstellung der Homeostase wird sie nach wenigen Tagen wieder entfernt und durch einen Fixateur externe oder eine innere Osteosynthese ersetzt.

Der Fixateur externe hat ein anderes Wirkungsprinzip. Hier werden Schanz'sche Schrauben in die Knochensubstanz des verletzten Beckens gebohrt. Die links und rechts angebrachten Schrauben werden dann über einen ventralen Fixateur oder Rahmen miteinander verbunden. Abhängig von der gewählten Konfiguration des Fixateur externe befinden sich die Schanz'schen Schrauben in der Beckenschaufel oder supraacetabulär. Biomechanische Studien haben nachgewiesen, dass ein ventral angebrachter Fixateur externe unabhängig von der Konfiguration niemals in der Lage ist, im hinteren Beckenring eine hohe Stabilität zu erzeugen. Hieraus ergibt sich, dass Patienten mit einem mechanisch komplett instabilen Beckenring, die nur mit einem ventralen Fixateur externe primär versorgt wurden, schlecht oder nicht aus dem Bett mobilisiert werden können. Für eine hohe Stabilität ist eine zusätzliche Osteosynthese des hinteren Beckenringes erforderlich.

Der Fixateur externe bietet eine nicht ausreichende Stabilität im hinteren Beckenring. Er wird als temporäre Maßnahme verwendet. Die definitive Versorgung erfordert manchmal eine zusätzli- 

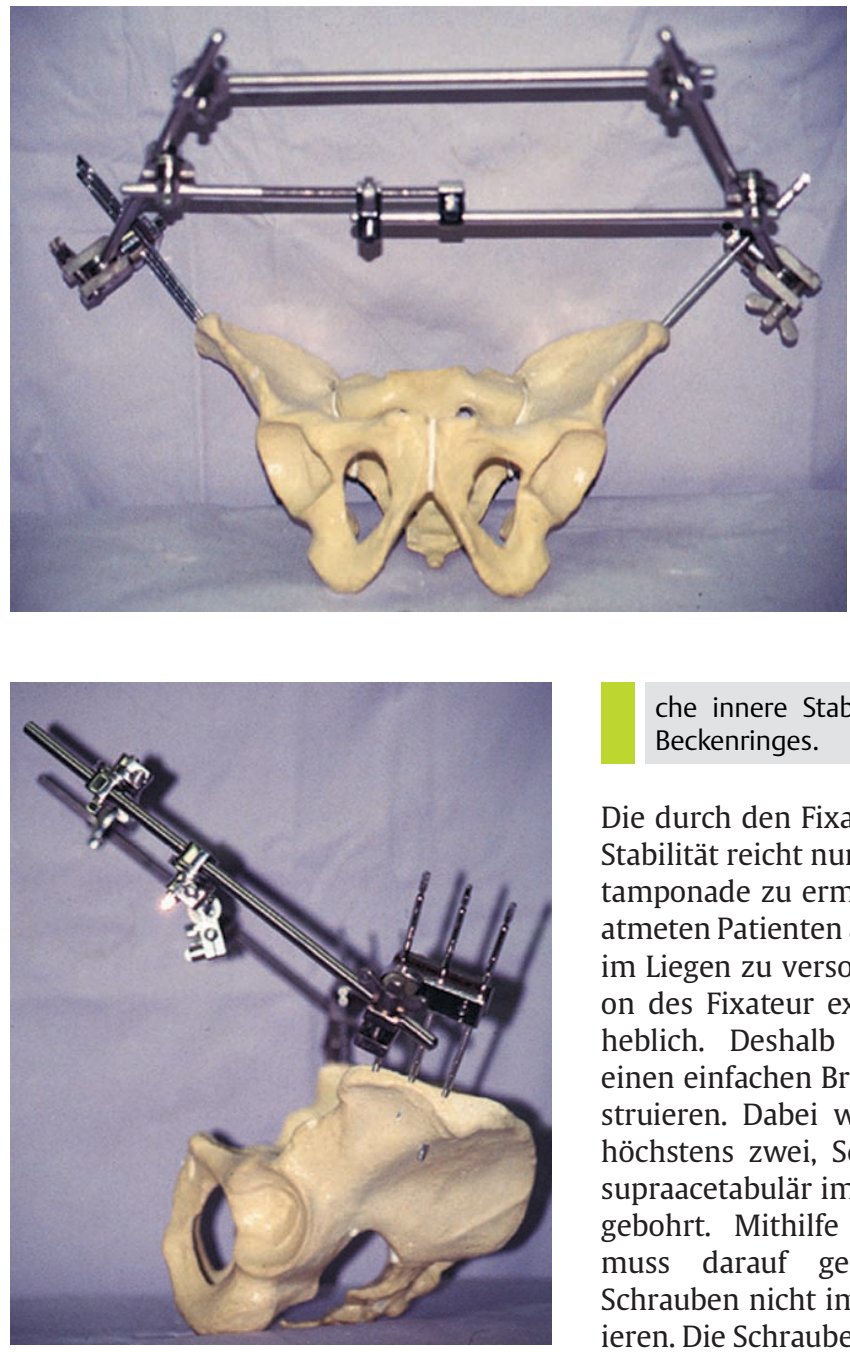

Abb.4b Seitenansicht.

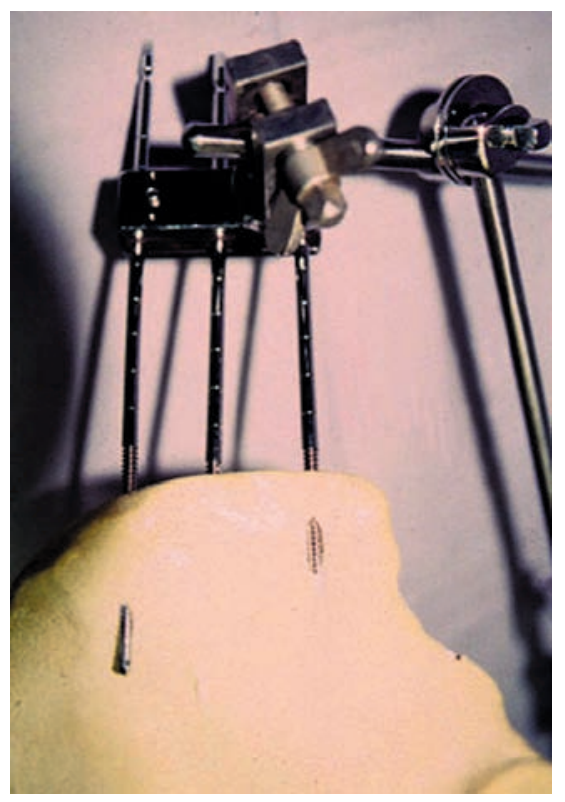

Abb.4c Die Detailansicht zeigt deutlich den kurzen Weg der Schrauben durch die dünne Beckenschaufel.
Abb. 4a Rahmenfixateur nach Slätis [9] mit je 3 Schanz'schen Schrauben in der linken und rechten BeckenschaufelFrontalansicht.

che innere Stabilisierung des hinteren Beckenringes.

Die durch den Fixateur externe erzeugte Stabilität reicht nur aus, um eine Beckentamponade zu ermöglichen und den beatmeten Patienten auf der Intensivstation im Liegen zu versorgen. Die Konfiguration des Fixateur externe ist dabei unerheblich. Deshalb bevorzugen wir es, einen einfachen Brückenfixateur zu konstruieren. Dabei wird jeweils nur eine, höchstens zwei, Schanz'sche Schrauben supraacetabulär im Corpus vom Os ilium gebohrt. Mithilfe der Durchleuchtung muss darauf geachtet werden, die Schrauben nicht im Hüftgelenk zu platzieren. Die Schrauben haben einen Winkel von etwa $40^{\circ}$ zur sagittalen Ebene und werden in Höhe der Spina iliaca anterior inferior von anterolateral nach posteromedial gebohrt. Sie kommen über einen Abstand von etwa $60 \mathrm{~mm}$ im Knochen $\mathrm{zu}$ liegen und erzeugen damit eine sehr hohe Ausreißfestigkeit (Abb.3a-d) [7]. Alternativ werden die Schrauben in die Beckenschaufel gebohrt. Die Ausreißfestigkeit dieser Schrauben ist allerdings viel geringer. Dies erklärt sich mit der Anatomie der Beckenschaufel. Viele Schrauben perforieren bereits nach kurzem Abstand die Tabula externa oder interna. Eine intraoperative Kontrolle der korrekten Schraubenlage mithilfe der Durchleuchtung ist viel schwieriger. Die Verbindungsstangen befinden sich auch viel weiter von dem Ort der Instabilität als bei dem supraacetabulären Brückenfixateur (Abb.4a-c) [9].

\section{Definitivversorgung}

Sowohl das Anbringen einer Beckenzwinge als auch das Platzieren eines Fixateur externe bei einem hämodynamisch

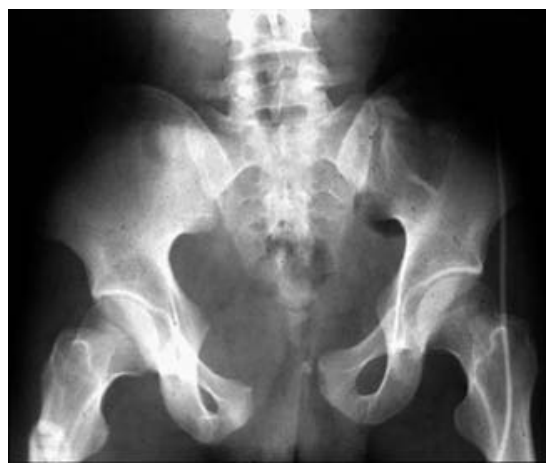

Abb.5a „Open-book“-Verletzung bei jungem Motorradfahrer.

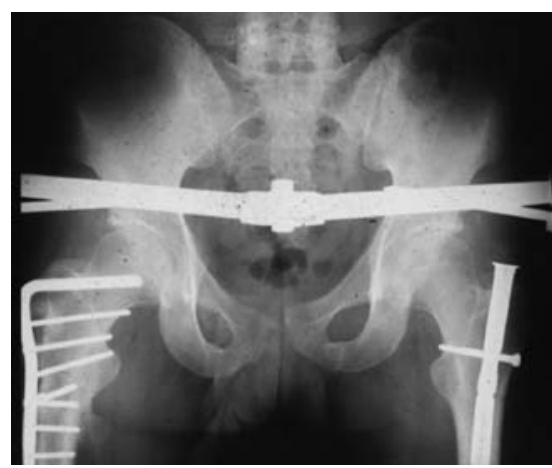

Abb.5b Versorgung der Beckenringverletzung mittels Brückenfixateur.

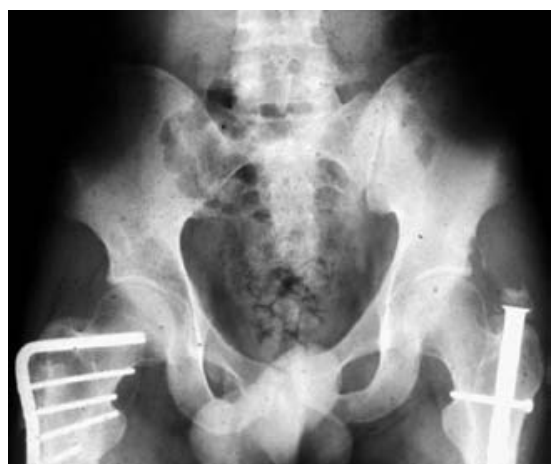

Abb.5c Zustand nach Abnahme des Fixateurs nach 6 Wochen. Die Verletzung wurde mit dem Fixateur zur Ausheilung gebracht.

instabilen Patienten werden als temporäre Maßnahmen betrachtet. Wenn der Patient das Trauma überlebt hat und ein stabiler hämodynamischer sowie homeostatischer Zustand erreicht wurde, hat die Behandlung der instabilen Beckenringverletzung andere Zielsetzungen. Nun geht es nicht mehr nur darum, einen stabilen Beckenring zu kreieren, sondern sowohl Anatomie und Funktion wiederherzustellen. Dazu muss ein symmetrischer und stabiler Beckenring rekonstruiert werden. Abhängig von der Art der Instabilität wird dafür eine Osteosynthese 


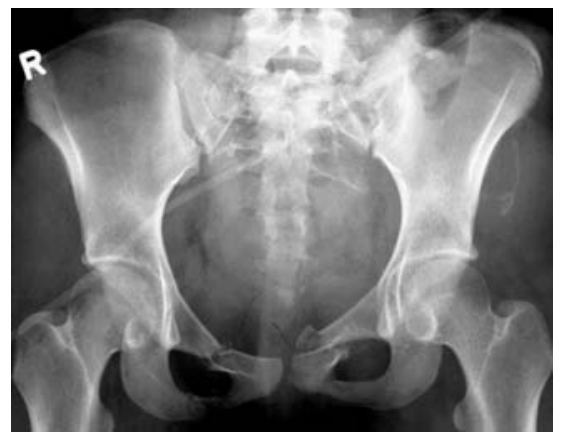

Abb.6a Auffahrunfall bei 17-jährigem Mädchen. Die Beckenübersichtsaufnahme zeigt eine bilaterale Sakrumverletzung mit Erweiterung des rechten Sakroiliakalgelenkes sowie bilaterale vordere Rami-Frakturen.
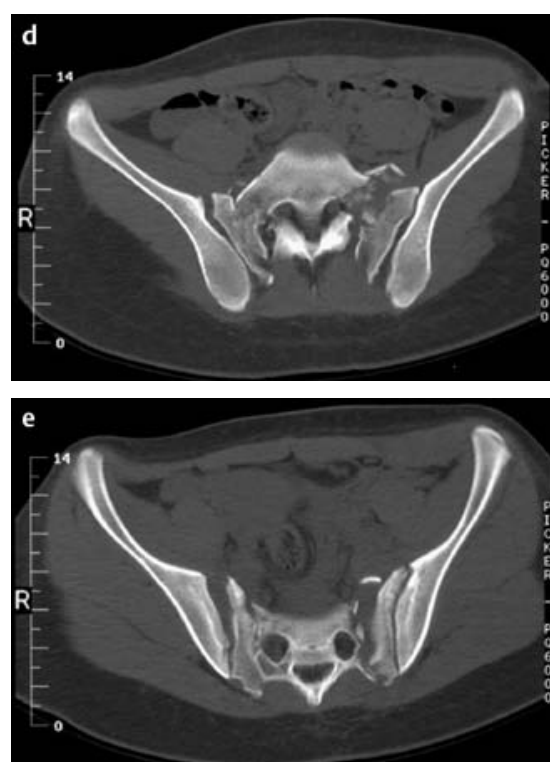

Abb.6 d, e CT-Schnitte in Höhe der Sakroiliakalgelenke.

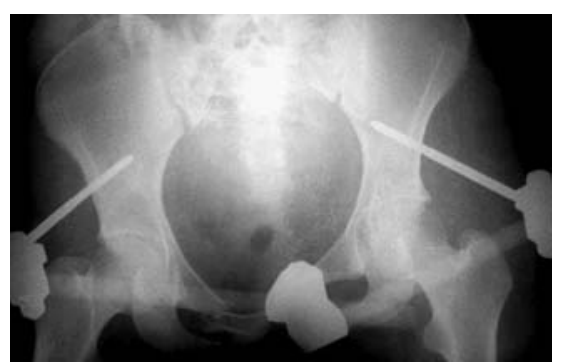

Abb.6f Anlage eines Fixateur externe bei hämodynamischer Instabilität.

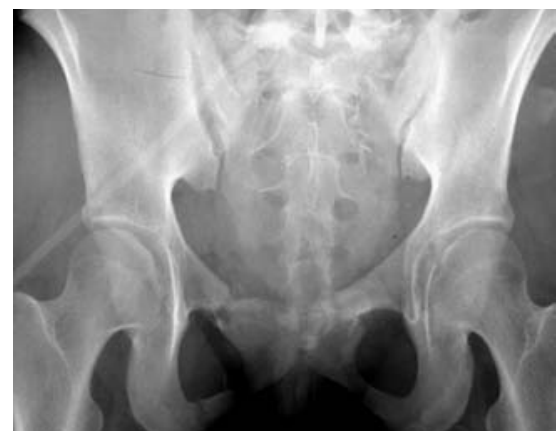

Abb.6b Beckeneingangsaufnahme.

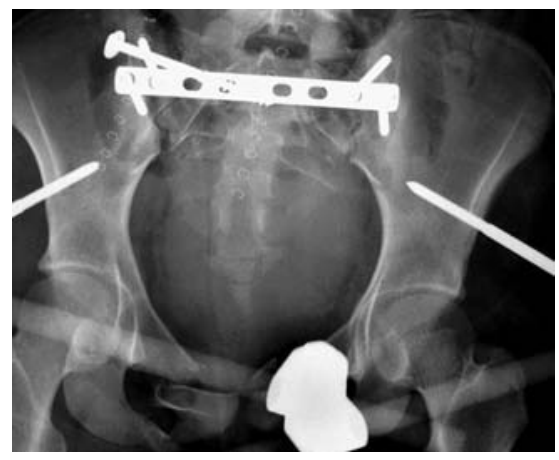

Abb.6g Definitivversorgung mittels überbrückender Plattenosteosynthese und sakroiliakaler Verschraubung rechts. Der Brückenfixateur wurde zur Stabilisierung der vorderen Beckeninstabilitäten beibehalten.

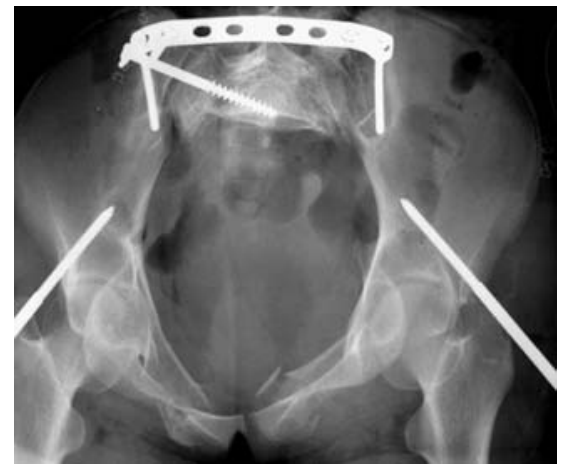

Abb.6h Beckeneingangsaufnahme.

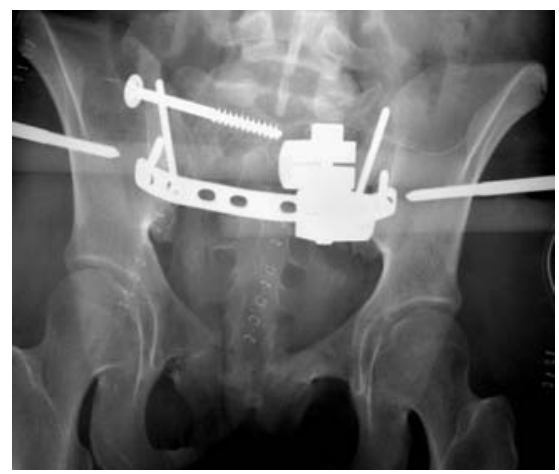

Abb.6i Beckenausgangsaufnahme.

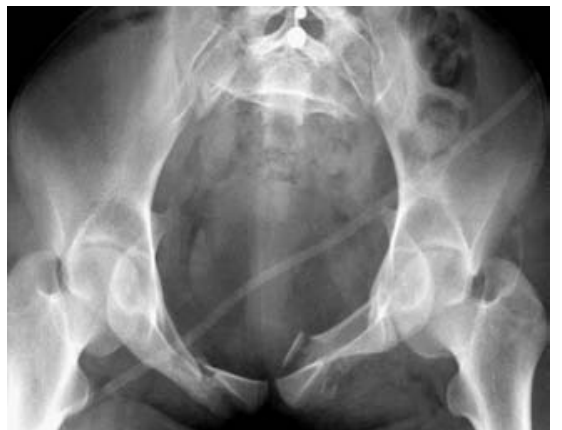

Abb.6c Beckenausgangsaufnahme.

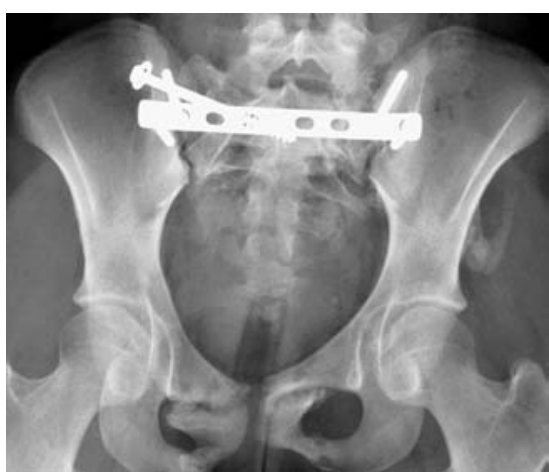

Abb.6j Zustand nach Entfernung des Fixateur externe.

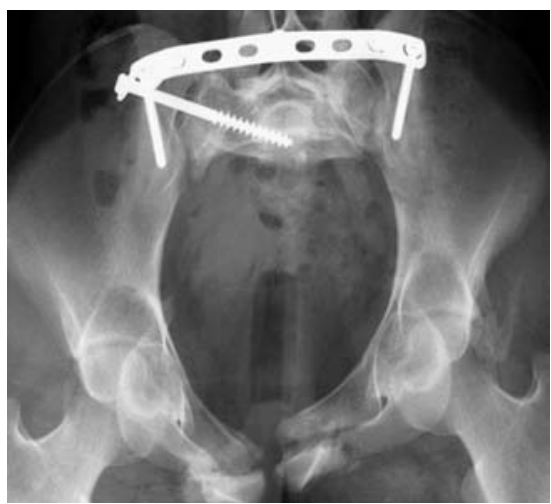

Abb.6k Beckeneingangsaufnahme.

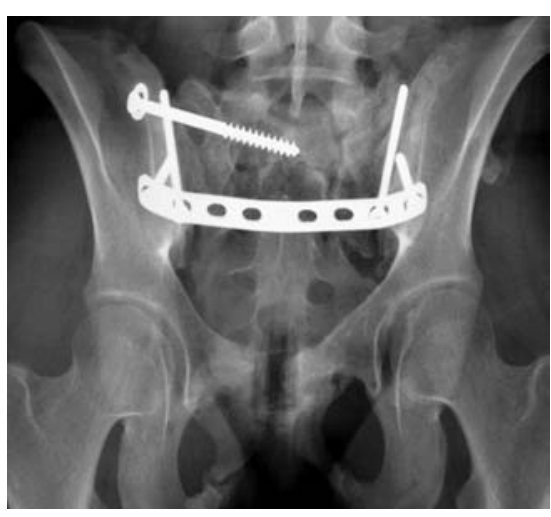

Abb.6I Beckenausgangsaufnahme. 
des hinteren und/oder des vorderen Beckenringes erforderlich sein. Die Art der Versorgung wird sich nach der Klassifikation der Verletzung richten, dem Alter des Patienten, nach dessen funktionellen Ansprüchen, Art und Ausmaß der begleitenden Weichteilverletzungen und nach der Erfahrung des behandelnden Teams [10].

\section{Eigene Erfahrungen}

In einem 9-Jahres-Zeitraum wurden 222 instabile Beckenringverletzungen operativ versorgt (8). Der Behandlungszeitraum lag zwischen 1986 und 1995, die Auswertungszeit zwischen 1995 und 2000. Es betraf 100 Verletzungen vom Typ B und 122 vom Typ C. Das Durchschnittsalter aller Patienten betrug zum Zeitpunkt des Unfalles 39,7 Jahre (zwischen 11,3 und 86,8 Jahre). Nur 13,1\% der Patienten wiesen keine Begleitverletzungen auf. 66\% der Patienten mit einer B-Verletzung wurden mit einem Fixateur externe erstversorgt. 52 Verletzungen (52\%) wurden mit dem Fixateur externe zur Ausheilung gebracht (Abb.5a-c). Die durchschnittliche Liegedauer des Fixateur externe betrug 53 Tage (zwischen 28 und 93 Tage). Ein Zweiteingriff wurde bei 15 B-Verletzungen (15\%) durchgeführt. Die Rate an Sekundäreingriffen lag bei den B1-Patienten bei $23 \%$, bei den B2/B3 Patienten bei 6,25\%. Der durchschnittliche Zeitraum zwischen Erstversorgung und Zweiteingriff betrug 8,9 Tage (zwischen 0 und 15 Tagen).

92 Patienten mit einer Beckenringverletzung vom Typ C $(75,4 \%)$ wurden mit einem Fixateur externe erstversorgt. 67 Verletzungen $(55 \%)$ wurden mit dem Fixateur externe zur Ausheilung gebracht. Die durchschnittliche Liegedauer des Fixateur externe betrug 56,1 Tage (zwischen 13 und 112 Tage). Ein Zweiteingriff wurde bei 32 Patienten (26,2\%) durchgeführt. Der durchschnittliche Zeitraum zwischen Erstversorgung und Zweiteingriff betrug 9,3 Tage (Abb. 6a-1).

Die Beckenringverletzungen vom Typ $C$ weisen eine höhere Mortalität und Morbidität auf. Die begleitenden Weichteilverletzungen führen häufiger zu funktionell schlechteren Endergebnissen.

Die Gesamtmortalität der 222 Patienten betrug $10,4 \%$. Die Mortalität beträgt in der B-Gruppe $5 \%$, in der C-Gruppe
14,8\%. 122 der 199 überlebenden Patienten $(61,3 \%)$ konnten nach einem durchschnittlichen Zeitraum von 21,6 Monaten klinisch und radiologisch nachuntersucht werden. Insgesamt hatten 34 der 55 nachuntersuchten Patienten mit einer Beckenringverletzung vom Typ B ein ausgezeichnetes Ergebnis (62\%), 11 Patienten hatten ein gutes (20\%), 9 ein mäßiges (16\%) und einer ein schlechtes (2\%) Resultat. Nur 23 der 67 nachuntersuchten Patienten mit einer Beckenringverletzung vom Typ $C$ hatten ein ausgezeichnetes Ergebnis (34,3\%), 25 ein gutes (37,3\%), 15 ein mäßiges (22,3\%) und 4 ein schlechtes $(6,0 \%)$. Die Beckenringverletzung vom Typ C stellt sich deshalb als viel gravierender als die des Typ B dar. Dies wird durch die viel höhere Mortalität, aber auch durch die schlechteren Endergebnisse deutlich. Patienten mit einer Beckenringverletzung vom Typ $\mathrm{C}$ weisen mehr begleitende Nervenläsionen und sonstige Weichteilverletzungen auf. Diese erholen sich in geringerem Maße als die begleitenden Verletzungen der B-Gruppe.

\section{Fazit}

Die mechanisch instabile Beckenringverletzung, die von einer hämodynamischen Instabilität begleitet ist, stellt eines der seltenen Traumen des muskuloskelettalen Systems dar, die schnell lebensbedrohlich werden können. Die Primärtherapie dieser Verletzung richtet sich sowohl auf das Einleiten einer aggressiven Schocktherapie nach den $A B C$-Regeln des ATLS als auch nach dem Beheben der mechanischen Instabilität. Die äußere Kompression auf den Beckenring wird in der Regel durch einen Fixateur externe, im Extremfall durch die Beckenzwinge realisiert. Beide Instrumente kennen ihre spezifischen Nachteile und Gefahren. Wenn mit dieser Maßnahme eine hämodynamische Stabilität nicht wiederhergestellt werden kann, bietet sich nur noch die selektive Arteriographie oder die Tamponade an. Die erste Prozedur kann nur nützlich sein, wenn eine arterielle Blutung vorliegt. Die Hämatomausräumung des kleinen Beckens mit Tamponade wird von uns bevorzugt, weil sie jede Art von Blutverlust vor Ort beherrschen kann. Die definitive Versorgung der Bekkenringverletzung richtet sich nach anderen Zielen als die Primärstabilisierung. Abhängig von der Art der Verletzung wird der Fixateur externe als ventraler Stabili- sator beibehalten und durch eine dorsale Osteosynthese ergänzt, alternativ kann eine ventrale und dorsal offene Reposition und innere Fixation durchgeführt werden.

\section{Literatur}

${ }^{1}$ Ben-Menachem Y, Coldwell DM, Young JWR, Burgess AR. Haemorrhage associated with pelvic fractures: causes, diagnosis and emergent management. Am J Roentgenol 1991; 157: 1005-1014

${ }^{2}$ Ertel W, Keel M, Eid K, Platz A, Trentz O. Control of severe haemorrhage using Cclamp and pelvic packing in multiply injured patients with a pelvic ring disruption. J Orthop Trauma 2001; 15: 468-474

${ }^{3}$ Ganz R, Krushell R, Jakob R, Küffer J. The antishock pelvic clamp. Clin Orthop 1991; 267: $71-78$

${ }^{4}$ Gercek E, Hessmann MH, Rommens PM. Bildgebende Verfahren bei Beckenverletzungen. Akt Traumatol 2002; 32: 163-170

5 Pohlemann T, Gännslen A, Bosch U, Tscherne $\mathrm{H}$. The technique of packing for control of haemorrhage in complex pelvic fractures. Tech Orthop 1995; 9: 267-270

${ }^{6}$ Pohlemann T, Paul C, Gännslen A, Tscherne $\mathrm{H}$. Die traumatische Hemipelvektomie. Erfahrungen aus 11 Fällen Unfallchirurg 1996; 99: 304-312

${ }^{7}$ Rommens PM, Hessmann MH. External fixation for the injured pelvic ring. Chapter 14 in: Fractures of the pelvis and acetabulum. Third Edition (M Tile, DL Helfet, JF Kellam, Eds) Lippincott Williams \& Wilkins, 2003 203-216

${ }^{8}$ Rommens PM, Hessmann MH. Staged reconstruction of pelvic ring disruption: differences in morbidirty, mortality, radiologic results and functional outcomes between B1, B2/B3 and C-type lesions. J Orthop Trauma 2002; 16: $92-98$

${ }^{9}$ Slätis P, Karaharju EO. External fixation of the pelvic girdle with a trapezoid compression frame. Injury 1975; 7: $53-58$

${ }^{10}$ Tscherne H, Pohlemann T. Becken und Acetabulum. Springer Berlin Heidelberg New York 1998

\section{Prof. Dr. med. Pol Maria Rommens} Chefarzt

Dr. med. Jens Heinermann

Assistenzarzt

Priv.-Doz. Dr. med. Martin Henri

Hessmann

Oberarzt

Klinik und Poliklinik für Unfallchirurgie Klinikum der Johannes Gutenberg Universität Mainz, Langenbeckstr. 1 D-55101 Mainz 